\title{
Interband absorption of photons by extended states in intermediate band solar cells
}

\author{
A. Luque , A. Mellor ，I. Ramiro , E. Antolín , I. Tobías , A. Martí
}

\begin{abstract}
A B S T R A C T
This paper considers sub-bandgap photon absorption in an InAs/GaAs quantum dot matrix. Absorption coefficients are calculated for transitions from the extended states in the valence band to confined states in the conduction band. This completes a previous body of work in which transitions between bound states were calculated. The calculations are based on the empirical k.p Hamiltonian considering the quantum dots as parallelepipeds. The extended states may be only partially extended-in one or two dimensions-or extended in all three dimensions. It is found that extended-to-bound transitions are, in general, weaker than bound-to-bound transitions, and that the former are weaker when the initial state is extended in more coordinates. This study is of direct application to the research of intermediate band solar cells and other semiconductor devices based on light absorption in semiconductors nanostructured with quantum dots.
\end{abstract}

\section{Introduction}

The intermediate band (IB) solar cell [1-3] is intended to increase the photocurrent of ordinary solar cells by forming a permitted band for electrons in the forbidden band (or bandgap) of a semiconductor. The increase of current is produced by photons that do not have enough energy to pump electrons from the valence band $(\mathrm{VB})$ the conduction band $(\mathrm{CB})$ can use the IB as a stepping stone, thus producing an increase in current.

IBs and IB cells have been prepared by several methods [4-6]; this paper is concerned with that in which the IB is formed by the bound state levels of a quantum dot (QD), usually of InAs in a GaAs matrix [7-14]. Sub-bandgap photocurrent has been measured [15] and a model has been developed able to reproduce approximately the internal quantum efficiency $[16,17]$.

This model proposes the so called empirical k · p Hamiltonian (EKPH) and is a four band $\mathrm{k} \cdot \mathrm{p}$ model [18] in which the spin orbit and strain induced terms are neglected in a first instance, but then they are accounted for by setting the measured band positions and effective masses in the eigenvalues of the four dimensional Hamiltonian matrix [16]. In this way, the eigenfunctions are of the form

$|\Xi(\mathbf{r})\rangle=|X\rangle \Psi_{x}(\mathbf{r})+|Y\rangle \Psi_{y}(\mathbf{r})+|Z\rangle \Psi_{z}(\mathbf{r})+|S\rangle \Psi_{s}(\mathbf{r})$.

where $|X\rangle,|Y\rangle,|Z\rangle$ and $|S\rangle$ are the exact Hamiltonian solutions for the
GaAs at the $I$ point, $|S\rangle$ corresponding to the $\mathrm{CB}$ and the others to the VB. Each solution may have positive or negative spin as the spin has not been considered in this solution. $\Psi_{x}(\mathbf{r}), \Psi_{y}(\mathbf{r}), \Psi_{z}(\mathbf{r})$, $\Psi_{s}(\mathbf{r})$ are the so called envolvent functions and may be considered as the projection of $|\Xi(\mathbf{r})\rangle$ on $|X\rangle,|Y\rangle,|Z\rangle$ or $|S\rangle$.

In this model, the dipole matrix elements for the transitions associated with photons are expressed as

$$
\begin{aligned}
\left\langle\Xi|\mathbf{u} \cdot \mathbf{r}| \Xi^{\prime}\right\rangle= & \left\langle\Psi_{x}|\mathbf{u} \cdot \mathbf{r}| \Psi_{x}{ }^{\prime}\right\rangle+\left\langle\Psi_{y}|\mathbf{u} \cdot \mathbf{r}| \Psi_{y}{ }^{\prime}\right\rangle+\left\langle\Psi_{z}|\mathbf{u} \cdot \mathbf{r}| \Psi_{z}{ }^{\prime}\right\rangle \\
& +\left\langle\Psi_{s}|\mathbf{u} \cdot \mathbf{r}| \Psi_{s}{ }^{\prime}\right\rangle
\end{aligned}
$$

where $\mathbf{u}$ is the polarization vector and $\mathbf{r}$ the position vector. The primes discriminate between the initial and final states.

This paper is organized as follows: after the introduction, a brief description is presented of the eigenvalues and eigenfunctions of the InAs in GaAs QDs classifying them and introducing their labeling. The results of the method of separation of variables to obtain the eigenvalues and the eigenfunctions are presented with a discussion of the limit of validity of this method and the modifications to be made. The calculation of the interband absorption coefficients are presented. Finally some conclusions are drawn. They also include a vision of the challenges ahead to obtain a practical QD IB solar cell.

\section{States and energy levels in quantum dot structured semiconductors}

The introduction of the InAs QDs into the GaAs matrix produces bound $(\mathrm{OE})$ states as represented in Fig. 1 . Some of these are 

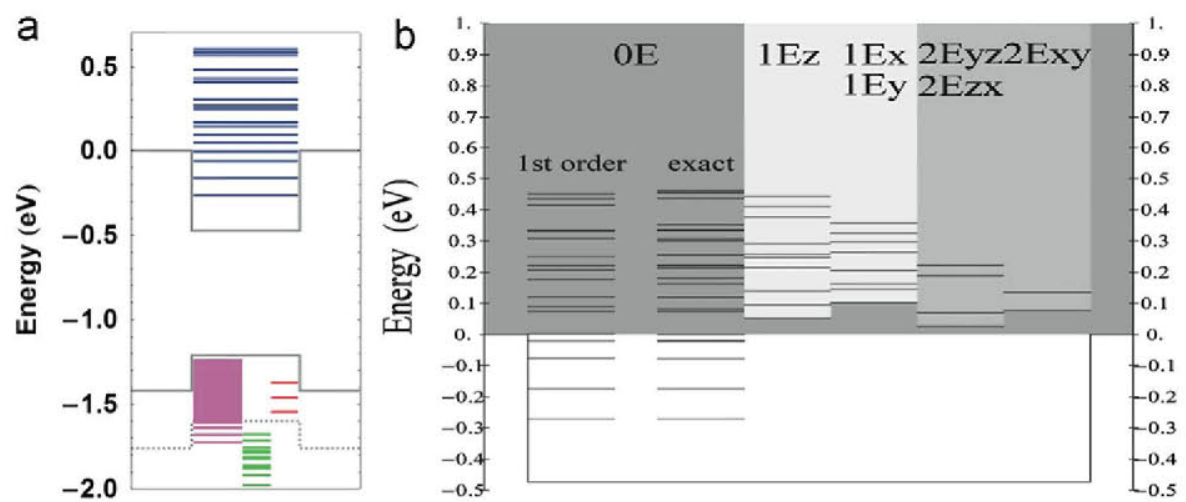

Fig. 1. (a) Energy levels of the OE bound states derived from the cb (blue) and from the hh (magenta), lh (red) and so (green) VBs calculated with the separation of variables approximation. (b) Energy levels of bound and extended states in the $\mathrm{CB}$ calculated according to an exact model (and a first order approximation model also for the OE states). The $0 \mathrm{E}$ are bound in all three dimensions, the $1 \mathrm{E}$ are bound in two dimensions and extended in the labeled dimension. The $2 \mathrm{E}$ states are bound in one dimension and extended in the labeled dimensions. Dark gray corresponds to 3E states extended in all three dimensions. (b) Reproduced from [19] with permission. (For interpretation of the references to color in this figure legend, the reader is referred to the web version of this article.)
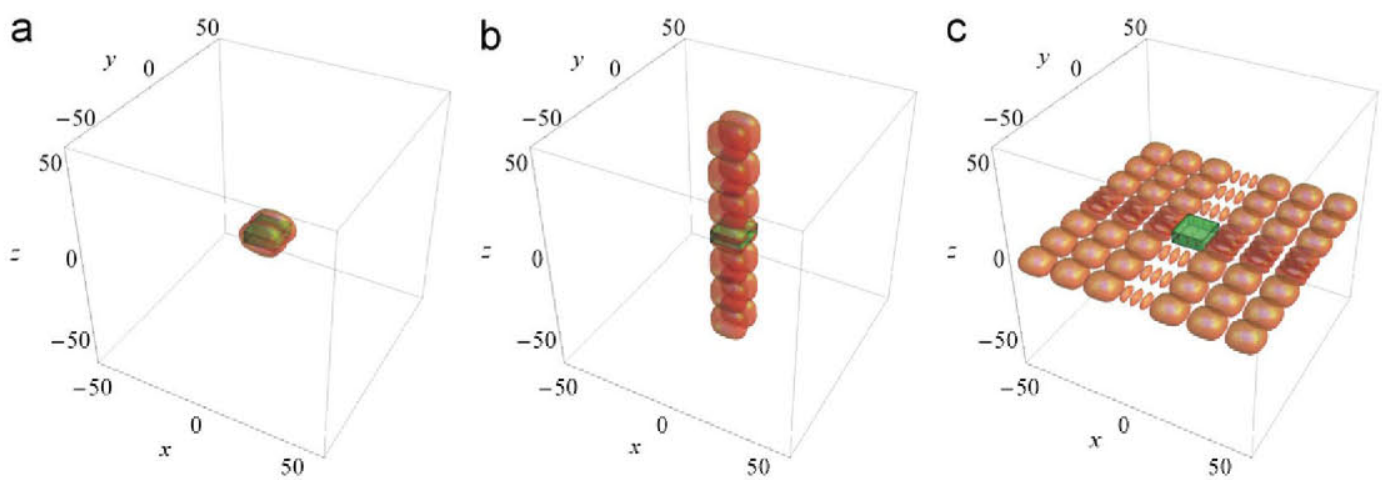

Fig. 2. Electron density-of-probability contours; space units in nanometer. (a) Bound $\mathrm{OE} \mathrm{cb}(1,2,1)$ wavefunction; (b) filamentary $1 \mathrm{Ez}$ cb(1,2,8) wavefunction; (c) sheet-shaped $2 \mathrm{Exy} \operatorname{cb}(5,8,1)$ wavefunction. Space units are in nanometer; the containing cube length is $2 L=120 \mathrm{~nm}$. The green box represents the $\mathrm{QD}$, of $16 \times 16 \times 6 \mathrm{~nm} \mathrm{~m}^{3}$. The contour drawn corresponds to 0.001 times the maximum density of probability of the $c b(1,1,1) \mathrm{OE}$ state. Figure reproduced from [19] with permission. (For interpretation of the references to color in this figure legend, the reader is referred to the web version of this article.)

derived from the CB (and they are generically called cb states) and some of them form the three bands that constitute the VB: heavy holes (hh) band, light holes (lh) band and spilt off (so) band. Some of these states are situated within the semiconductor bandgap and some are inside the $\mathrm{CB}$ and the $\mathrm{VB}$. The latter are called virtual bound states. In addition, there are extended states (3E) [19], with energy fully inside the $\mathrm{CB}$ or the $\mathrm{VB}$ as well as states that are extended in one $(1 \mathrm{E})$ or two $(2 \mathrm{E})$ dimensions and bound in the others. These have their energy also inside the $\mathrm{CB}$ or the $\mathrm{VB}$. Examples of wavefunctions representing bound states and partially extended sates are represented in Fig. 2.

To further clarify the nomenclature, blue levels in Fig. 1 are labeled $\mathrm{cb}(111), \mathrm{cb}(121)$, etc. for reasons explained later. The lower energy state $\mathrm{cb}(111)$ is the intermediate band (IB). If the semiconductor is n-doped, the electrons delivered will fall into this level partially filling it. Some photofilling is also possible as result of a detailed balance calculation $[20,21]$ which is beyond the scope if this paper. The remaining (blue) bound sates within the forbidden band are called ladder states (LS) [3] because they allow a more or less strong thermal contact between the IB and the $\mathrm{CB}$.

From now on, the term $\mathrm{CB}$ shall only refer to states with energy above the GaAs CB bottom. Although the IB and LS are formed from the $\mathrm{CB}$ of the heterostructure, they are detached from the host $\mathrm{CB}$ and hence we use the lowercase nomenclature $\mathrm{cb}(121)$, etc. to describe them. On the contrary, the VB will include not only the states with energy below the GaAs VB top but also the magenta and green states even if they are within the GaAs bandgap. The reason for this is that they are expected to have the same quasi-Fermi level than all the states within the GaAs VB. It comprises all the hh, lh and so states.

\section{The separation of variables model}

In the EKPH model, the calculation of the energy levels is easy. The effective mass time-independent Schrödinger equation (TISE)

$H \Phi=-\frac{\hbar^{2}}{2 m^{*}} \nabla^{2} \Phi+V(\mathbf{r}) \Phi=E \Phi$

is to be solved for each band; $m$ is the effective mass and $V(\mathbf{r})$ is the band offset due to the inhomogeneity of the material. In the VBs, the effective masses are negative. Thus potential pedestals in the VBs behave as wells. These equations will give the set of energies. In our calculations, we have considered a very simple $V(\mathbf{r})$ function that is a "squared potential well/pedestal" in all the QD volume.

In the solution of the TISE, a separation of variables approximation has been applied so that $\Phi(\mathbf{r})=\xi(x) \Psi(y) \zeta(z)$. Each one of these solutions is the solution of a one dimensional (1D) TISE, e.g.

$\frac{\hbar^{2}}{2 m^{*}} \frac{\nabla^{2} \xi}{\xi}+E_{x}= \begin{cases}0 & \forall|x|<a \\ U & \forall|x| \geq a\end{cases}$

$U$ being the band offset and $2 a$ the length of the QD in the $x$ direction. The same applies to $\psi(y)$ and $\zeta(z)$.

Finding the solutions for $\xi(x)$ (or for $\psi(y), \zeta(z)$ ) constitutes a simple exercise of differential equations. In this context, a 
discussion is provided e.g. in [22]. For $E_{x}<U$, bounded solutions, different from the trivial $\xi(x) \equiv 0$, are even $(\cos (k x))$ or odd $(\sin (k x))$ harmonic functions inside the well flanked by fading exponential functions outside it $\left(\exp \left(-\kappa_{x} x\right) \forall x \geq a\right)$. Solutions may only exist for certain values of the wavenumbers $k_{n}$ for which the non-fading exponential solution is canceled. The energy $E_{n}$, fading coefficient $\kappa_{n}$ and $k$-values are related by

$E_{n}=\hbar^{2} k_{n}^{2} / 2 m^{*}=U-\hbar^{2} \kappa_{n}^{2} / 2 m^{*}$

The index $n$ denotes the different permitted energies in increasing order. It is a quantum number $(\mathrm{QN})$. Odd QNs correspond to even functions and vice versa.

For $E \geq U$, the solution is harmonic with wavenumber $k$ inside the potential well and also harmonic, even or odd, outside it but with a different value of the wavenumber $k_{e}$ and a phase term. That is, they are of the form $\cos \left(k_{e} x-\theta\right)$ or $\sin \left(k_{e} x-\theta\right)$. Details can be found, e.g. in [23]. In this case,

$E_{n}=U+\hbar^{2} k_{e}^{2} / 2 m^{*}=\hbar^{2} k^{2} / 2 m^{*}$

and

$\left(k_{e} / k\right) \cot (k a)=\cot \left(k_{e} a-\theta\right)$

$\left(k_{e} / k\right) \tan (k a)=\tan \left(k_{e} a-\theta\right)$

respectively for the even and odd functions.

For $E \geq U, k_{e}$ can take any value and therefore it leads to a continuum spectrum of energies. Since the mathematics of continuum spectra is rather complicated, it is common to assume that the wavefunctions are restricted to a large but finite region (a segment of length $2 L$, with large $L$, for one-dimensional cases, or a big parallelepiped for three-dimensional ones) and assume periodic conditions there. This leads [23] to

$k_{e} L-\theta=\tilde{n} \pi / 2$

where $\tilde{n}$ is an integer, odd for the even solutions and even for the odd solutions.

Neglecting the variation of $\theta$, the permitted values of $k_{e}$ are separated by $\Delta k_{e} \cong \pi / 2 L$ (or $\Delta k_{e} \cong \pi / L$ for states of the same parity), which is small as long as $L$ is big (with respect to $a$ ). However, only a numerable set of $k_{e}$-values are now permitted and the new $\mathrm{QN}$, $\tilde{n}$, has appeared. Nevertheless, in most cases in this paper, we will use $k_{e}$ rather than $\tilde{n}$.

As already advanced, the three dimensional (3D) TISE solutions are labeled, within this approximation, as $\mathrm{cb}(111), \mathrm{hh}(121)$, etc., the triplets of integers referring to the QNs of the $1 \mathrm{D}$ solutions. Their energy is

$E_{n_{x}, n_{y}, n_{y}}=E_{x, n_{x}}+E_{y, n_{y}}+E_{z, n_{z}}$

These energies, within this approximation, are represented in Fig. 1(a) for all the bands. The hh has many levels and in the graphic they appear as a quasi continuum. These values correspond to the parameters in Table 1 which are applicable to the QDs described and measured (as sample SB) in [15].

The validity of the separation of variables approximation has been examined in [19]. They are not exact eigenvalues of the Hamiltonian $H$ but they form a complete orthogonal set of eigenfunctions. By developing the Hamiltonian in these eigenfunctions and diagonalizing the obtained matrix, the exact eigenvalues are found. For the case of the $\mathrm{CB}$ they are represented in Fig. 1(b).

Table 1

Parameters used in the calculations.

\begin{tabular}{llllll}
\hline$a(\mathrm{~nm})$ & 8 & $m_{l h} / m_{e}$ & 0.333 & $U_{v b}(\mathrm{eV})$ & 0.212 \\
$c(\mathrm{~nm})$ & 3 & $m_{\text {so }} / m_{e}$ & 0.076 & $U_{s o}(\mathrm{eV})$ & 0.339 \\
$m_{c b} / m_{\mathrm{e}}$ & 0.0294 & $E_{\mathrm{g}}(\mathrm{eV})$ & 1.42 & $E_{s o} \mathrm{GaAs}(\mathrm{eV})$ & 0.341 \\
$m_{l h} / m_{e}$ & 0.027 & $U_{c b}(\mathrm{eV})$ & 0.473 & & \\
\hline
\end{tabular}

The energies obtained with the separations of variables approximation are very close to the exact ones for the bound states within the bandgap and for the lowest virtual bound states (below $0.2 \mathrm{eV}$ within the $\mathrm{CB}$ ) but after this the energy levels are pushed down, considerably for the highest energies. The first order approximation energies are also shown for bound states in Fig. 1 (b). They are rather close to the exact ones. In this paper, we use this approximation, obtained by adding $\left\langle\Phi\left|V^{\prime}\right| \Phi\right\rangle$ to the energy obtained in the zero order (separation of variables), where $V^{\prime}$ is the difference between the Hamiltonian and the one admitting as solutions those with separation of variables (see its expression in [19]).

The energy of an absorbed photon is the difference between the final and initial electronic state energies. We call it $E_{\text {line. }}$. In this case it is obtained by subtracting the VB extended state energy, calculated to the 1 st order approximation, from the $\mathrm{CB}$ bound state energy within the bandgap, calculated in the separation of variables approximation (further approximation makes no significant change). That is,

$E_{\text {line }}=E_{G}+E_{B S c b}-U_{v b}+\left(\frac{\hbar^{2}\left(k_{n_{x}}^{2}+k_{n_{y}}^{2}+k_{n_{z}}^{2}\right)}{2 m^{*}}\right)+\left\langle\Phi\left|V^{\prime}\right| \Phi\right\rangle$

where $E_{B S C b}$ is the cb bound state, which is negative for the cases in study (as well as $\left\langle\Phi\left|V^{\prime}\right| \Phi\right\rangle$ ).

According to Eq. (6), in the case of extended states, these equations are

$$
\begin{aligned}
& \left\{\begin{array}{l}
E_{z(n x, n y)}=E_{G}+E_{B S c b}+\left(\frac{\hbar^{2}\left(k_{x x}^{2}+k_{n y}^{2}\right)}{2 m^{*}}\right)+\left\langle\Phi\left|V^{\prime}\right| \Phi\right\rangle+\left(\frac{\hbar^{2} k_{e z}^{2}}{2 m^{*}}\right) \\
=E_{T z(n x, n y)}+\left(\frac{\hbar^{2} k_{e z}^{2}}{2 m^{*}}\right)
\end{array}\right. \\
& \left\{\begin{array}{l}
E_{x y(n z)}=E_{G}+E_{B S c b}+U_{v b}+\left(\frac{\hbar^{2} k_{n z}^{2}}{2 m^{*}}\right)+\left\langle\Phi\left|V^{\prime}\right| \Phi\right\rangle+\left(\frac{\hbar^{2}\left(k_{e x}^{2}+k_{e y}^{2}\right)}{2 m^{*}}\right) \\
=E_{T x y(n z)}+\left(\frac{\hbar^{2}\left(k_{e x}^{2}+k_{e y}^{2}\right)}{2 m^{*}}\right)
\end{array}\right. \\
& \left\{\begin{array}{l}
E_{x y z}=E_{G}+E_{B S c b}+2 U_{v b}+\langle\Phi|H| \Phi\rangle+\left(\frac{\hbar^{2}\left(k_{e x}^{2}+k_{e y}^{2}+k_{e z}^{2}\right)}{2 m^{*}}\right) \\
=E_{G}+E_{B S c b}+\left(\frac{\hbar^{2}\left(k_{e x}^{2}+k_{e y}^{2}+k_{e z}^{2}\right)}{2 m^{*}}\right)=E_{T x y z}+\left(\frac{\hbar^{2}\left(k_{e x}^{2}+k_{e y}^{2}+k_{e z}^{2}\right)}{2 m^{*}}\right)
\end{array}\right.
\end{aligned}
$$

for $1 \mathrm{E}, 2 \mathrm{E}$ and $3 \mathrm{E}$ states respectively. The subindices clarify which variables are extended and which are bound. QNs always refer to bound variables. The formulas for other extended variables are obtained by permuting the variables. In all the cases the continuous spectrum appears above a certain threshold that depends on the energy of the confined part of the eigenfunction. This is clearly visible in Fig. 1(b) where the lines in the regions $1 \mathrm{E}$ and $2 \mathrm{E}$ represent these thresholds. Above one of them there is a continuum of states with one or two $k_{e}$ variables that can be freely chosen. However what is reported in this figure corresponds to the $\mathrm{CB}$, while in this paper we are interested in the extended states in the VB.

Notice that every extended dimension produced adds $U_{v b}$ to the threshold energy which is partly compensated by the negative value of $\left\langle\Phi\left|V^{\prime}\right| \Phi\right\rangle$ so that the largest threshold energies approach to the top of the $\mathrm{VB}$ (the smallest threshold energies approach to the bottom of the CB in Fig. 1(b)). In the 3E case, $\left\langle\Phi\left|V^{\prime}\right| \Phi\right\rangle=-2 U_{v b}$, so that the two terms balance out. This means that the threshold for $3 \mathrm{E}$ functions in the $\mathrm{VB}$ is the top of it.

In this paper we are using a first order approximation approach. The use of exact values is not convenient because the wavefunctions are then linear combinations of separation-ofvariables wavefunctions (which is a basis), in most cases with one dominant basis function. This makes the labeling of the states cumbersome. Also, the absorption lines would be a mixture of two or more of the absorption coefficients used here, although with 
one dominating. Thus the use of the exact wavefunctions makes the calculations inextricable. The use of the first order approximation renders the problem manageable.

\section{Calculation of the absorption coefficients}

The absorption coefficient for transitions between levels under vertical $(z)$ illumination is given by [16]

$$
\begin{aligned}
\alpha_{v, \text { line }}(E) & =2 \frac{2 \pi^{2} e^{2} E}{n c h \varepsilon_{0}} \frac{\left[\left|\left\langle\Xi|x| \Xi^{\prime}\right\rangle\right|^{2}+\left|\left\langle\Xi|y| \Xi^{\prime}\right\rangle\right|^{2}\right] / 2}{4 a^{2}} F N_{l} \delta\left(E-E_{\text {line }}\right) \\
& =\alpha^{\prime} E \delta\left(E-E_{\text {line }}\right)
\end{aligned}
$$

where $n$ is the index of refraction, $F$ is the fraction of surface covered by QDs, and $N_{l}$ is the number of QD layers per centimeter. $E_{\text {line }}$ is the energy difference between the initial and final states. The rest are universal constants with their usual symbols.

We present in Fig. 3 (in red) the sub-bandgap absorption for transitions between bound states (including the virtual bound states) under vertical illumination. Under the hypothesis of full collection of the generated electron-hole pairs, this absorption has permitted calculation of the internal quantum efficiency. It has agreed rather well with the measured value $[16,17]$. This absorption has been calculated by calculating all the corresponding envolvents and employing Eqs. (2) and (12). For the calculation of the envolvents, the procedure as described in $[16,17]$ comprises four steps: (1) calculate the (initial or final) state eigenfunctions using the corresponding effective mass Eq. (2) obtain its Fourier transform; (3) multiply it by the appropriate element of the transformation matrix; (4) obtain its inverse Fourier transform. The transformation matrix is formed of a four dimensional square matrix whose rows correspond to the different bands ( $\mathrm{cc}, \mathrm{lh}$, hh, so) and whose columns correspond to the possible projections $(|X\rangle,|Y\rangle,|Z\rangle,|S\rangle)$. The Fourier transforms are discrete Fourier transforms in the usual case of numerical calculations. The total absorption is obtained as the sum of the absorption coefficients for all the lines between couples of bound states in the VBs and in the $\mathrm{CB}$, limiting our calculations to the energies below the bandgap. The Dirac deltas appearing in Eq. (12) are drawn as Gaussian functions to account for the slight variations of level positions due e.g. to size variations of the QDs. The same procedure will be used in this paper for the calculation of the transitions between the different types of extended states in the

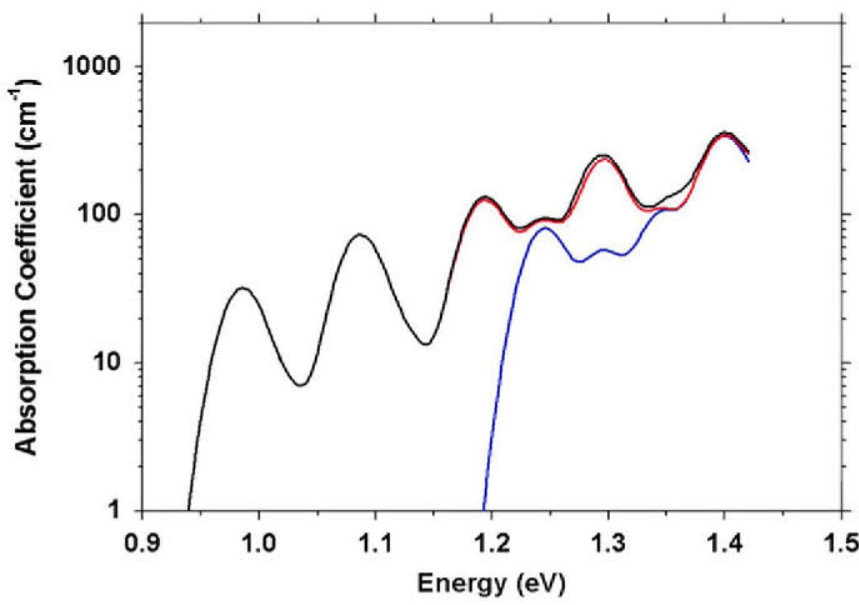

Fig. 3. Red: total sub-bandgap absorption due to transitions between bound states. Blue: sub-bandgap absorption between VB bound states and virtual bound states within the $\mathrm{CB}$. Black: total sub-bandgap absorption due to the former transitions and transitions between bound and extended states. (For interpretation of the references to color in this figure legend, the reader is referred to the web version of this article.)
VBs and the IB and ladder states. However the continuous nature of the extended states implies substitution of the sums by integrals.

We study now the absorption coefficients for the transitions from $1 \mathrm{E}, 2 \mathrm{E}$ and $3 \mathrm{E}$ VB states to the $\mathrm{IB}$ and ladder states. We take as examples the transitions $\mathrm{hh}(31 e) \rightarrow \mathrm{cb}(121), \mathrm{lh}(e 2 o) \rightarrow \mathrm{cb}(111)$ and $\mathrm{hh}($ eoe $) \rightarrow \mathrm{cb}(221)$. The labels $e$ and $o$ refer to even or odd one 1D extended states. The expression for the $1 \mathrm{E}, 2 \mathrm{E}$ and $3 \mathrm{E}$ cases are respectively,

$\alpha_{h h(31 e) c b(121)}=\frac{L}{\pi} \int_{0}^{\infty} \alpha_{h h(31 e) c b(121)}^{\prime}(E) E \delta\left(E-E_{\text {line }}\right) \frac{d k_{z}}{d E} d E$

$\alpha_{\text {lh }(e 20) c b(111)}=\frac{L^{2}}{\pi^{2}} \int_{0}^{\pi / 2} d \omega \int_{0}^{\infty} \alpha_{l h(e 20) c b(111)}^{\prime}(E, \omega) E \delta\left(E-E_{\text {line }}\right) \frac{d\left(k_{x}, k_{z}\right)}{d(E, \omega)} d E$

$\alpha_{h h(e o e) c b(221)}=\frac{L^{3}}{\pi^{3}} \int_{0}^{\pi / 2} d \varphi \int_{0}^{\pi / 2} d \theta \int_{0}^{\infty} \alpha_{h h(e o e) c b(221)}^{\prime} E \delta\left(E-E_{\text {line }}\right) \frac{d\left(k_{x}, k_{y}, k_{z}\right)}{d(E, \theta, \varphi)} d E$

where we have made the following the transformations of variables, respectively.

$\left\{k_{e z}=\left(2 m^{*} / \hbar^{2}\right)^{1 / 2}\left(E-E_{T z(n x, n y)}\right)^{1 / 2}\right.$

$\int k_{e x}=\left(2 m^{*} / \hbar^{2}\right)^{1 / 2}\left(E-E_{T x y(n x)}\right)^{1 / 2} \cos \omega$

$\left\{k_{e y}=\left(2 m^{*} / \hbar^{2}\right)^{1 / 2}\left(E-E_{T x y(n x)}\right)^{1 / 2} \sin \omega\right.$

$\left\{\begin{array}{l}k_{x}=\left(2 m^{*} / \hbar^{2}\right)^{1 / 2}\left(E-E_{T x y z}\right)^{1 / 2} \cos \varphi \sin \theta \\ k_{y}=\left(2 m^{*} / \hbar^{2}\right)^{1 / 2}\left(E-E_{T x y z}\right)^{1 / 2} \sin \varphi \sin \theta \\ k_{z}=\left(2 m^{*} / \hbar^{2}\right)^{1 / 2}\left(E-E_{T x y z}\right)^{1 / 2} \sin \varphi \sin \theta\end{array}\right.$

The Jacobians are,

$\frac{d k_{z}}{d E}=\sqrt{\frac{m^{*}}{2 \hbar^{2}\left(E-E_{T z(n x, n y 1)}\right)}}$

$\frac{d\left(k_{z}, k_{y}\right)}{d(E, \omega)}=\left(\frac{m^{*}}{\hbar^{2}}\right)$

$\frac{d\left(k_{z}, k_{y}, k_{z}\right)}{d(E, \theta, \varphi)}=\left(\frac{2^{1 / 3} m^{*}}{\hbar^{2}}\right)^{3 / 2} \sin \theta\left(E-E_{T x y z}\right)^{1 / 2}$

So that the absorption coefficients result

$\left\{\alpha_{h h(31 e) c b(121)}=\frac{L}{h \pi} \sqrt{\frac{m_{h h}}{2}} \frac{E \alpha_{h t h(31 \mathrm{e}) c b(121)}^{\prime}\left(E_{\text {line }}\right)}{\sqrt{\left(E_{\text {line }}-E_{T z(31)}\right)}}\right.$

$\left\{\alpha_{l h(e 20) c b(111)}=\left(\frac{m_{l h} L^{2}}{\pi^{2} \hbar^{2}}\right) E_{l i n e} \int_{0}^{\pi / 2} \alpha_{l h(e 2 o) c b(111)}^{\prime}(E, \omega) d \omega\right.$

$\left\{\alpha_{h h(e o e) c b(221)}=L^{3}\left(\frac{2^{1 / 3} m_{h h}}{\pi^{2} \hbar^{2}}\right)^{3 / 2}\left(E_{\text {line }}-E_{T x y z}\right)^{1 / 2} E_{\text {line }} \int_{0}^{\pi / 2} d \varphi \int_{0}^{\pi / 2} \alpha_{h h(e e e) c b(221)}^{\prime} \sin \theta d \theta\right.$

Using the averaged values of the elementary transition absorption coefficients (from now on $E_{\text {line }}$ will be written $E$ for brevity)

$\hat{\alpha}_{\ln (e 20) c b(111)}^{\prime}(E)=\frac{2}{\pi} \int_{0}^{\pi / 2} \alpha_{\ln (e 2 o) c b(111)}^{\prime}(E, \varphi) d \varphi$

$\widehat{\alpha}_{h h(e o e) c b(111)}^{\prime}(E)=\frac{2}{\pi} \int_{0}^{\pi / 2} d \varphi \int_{0}^{\pi / 2} \alpha_{h h(e o e) c b(111)}^{\prime}(E, \theta, \varphi) \sin \theta d \theta$

we can write

$\alpha_{h h(31 e) c b(121)}=\frac{L}{\hbar \pi} \sqrt{\frac{m_{h h}}{2}} \frac{E \alpha_{h h(31 e) c b(121)}^{\prime}(E)}{\sqrt{\left(E-E_{T z(31)}\right)}}$

$\alpha_{l h(e 20) c b(111)}=\left(\frac{L^{2} m_{l h}}{2 \hbar^{2} \pi}\right) E \hat{\alpha}_{l h(e 2 o) c b(111)}^{\prime}(E)$

$\alpha_{h h(e o e) c b(221)}=L^{3}\left(\frac{m^{*}}{2^{1 / 3} \hbar^{2} \pi^{4 / 3}}\right)^{3 / 2}\left(E-E_{T x y z}\right)^{1 / 2} E \hat{\alpha}_{h h(e o e) c b(221)}^{\prime}(E)$ 
Notice that below the threshold energy no absorption is produced. For the $1 \mathrm{E}$ states this absorption coefficient has a singularity (infinite) in $E_{T z(31)}$, similar to that in the quantum wires. As done with the Dirac deltas for the bound states, here this singularity is smoothed with a soft step function.

To obtain all the absorptions form the $1 \mathrm{E}$ functions to the $\mathrm{cb}$ (121) bound state we must add all the combinations of $(n x, n y, e)$ and then ( $n x, n y, 0)$ with $n x, n y$, running form $1-7$. The symmetries may reduce number of calculations (approximately divided by 8 ) because $x$ and $y$ are symmetric and the parity either $n x$ or $n y$ has to differ once in the initial and final states [17]. To this we must sum the absorptions for the 1Ey where the quantum numbers are $(n x, e / o, n z)$ and the same with $1 \mathrm{Ex}$.

Similar treatment has to be done with the lh band, adding again the resulting curves. In principle the same should be done with the so band but its energy position is too low and the transitions fall beyond the bandgap, where we are not interested.

Similar sums have to be done with the $2 \mathrm{E}$ states and with the $3 \mathrm{E}$ states (in this latter case all the combinations of parities have to be summed up).

The global results are presented for all the $1 \mathrm{E}, 2 \mathrm{E}$ and $3 \mathrm{E}$ as initial states to the final states $c b(111), c b(121)$ (including the degenerate $c b(211))$ and $c b(221)$ in Fig. 4. In this calculation, the IB has been considered empty so presenting higher absorption. The red curves represent the transitions between bound states, as calculated in [17], blue, green and purple curves correspond to the $1 \mathrm{E}, 2 \mathrm{E}$ and $3 \mathrm{E}$ initial states. It can be seen that the absorptions from the $1 \mathrm{E}$ states tend to be dominant only for photons close to the bandgap energy. Furthermore, $2 \mathrm{E}$ absorptions are smaller and $3 \mathrm{E}$ absorptions are negligible. The onset of the absorption coefficient is located in all cases near the $\mathrm{VB}$ top. In the $3 \mathrm{E}$ case this is strictly at the VB top.

The absorption from VB extended states and $\mathrm{CB}$ virtual bound states is not calculated in this paper because it leads to transitions beyond the bandgap range of energies.

\section{Conclusions}

The most straightforward conclusion of this work is represented in Fig. 4. It can be concluded that the extended states absorb less light than the bound states. This is intuitive because the occurrence of a transition requires an overlap of the initial and final states. When one of these is extended, then the overlap is reduced; this is partly compensated by the existence of many extended sates, but not sufficiently to give strong absorption, as demonstrated in this article. $1 \mathrm{E}$ extended states lead to higher absorption than $2 \mathrm{E}$ and much more than $3 \mathrm{E}$ but still smaller than OE states. Spherical QDs do not have virtual bound states (see e.g. [24] chap. 11). Therefore, transitions from the cb bound sates within the bandgap and the CB may be too weak for spherical QDs.

The curves in Fig. 4 provide data for an exact detailed balance calculation of the intermediate band solar cell, like the one in [21].

In this paper, only absorptions within the bandgap range of energies are calculated. The reason for not considering photons beyond the bandgap is that the light reaching the IB material is filtered by the emitter so that no photon with energy above the bandgap reaches the IB material. Even if some reach it, they will be mainly absorbed by the strong absorption between extended states of the VB and the $\mathrm{CB}$.

The sub-bandgap absorption by transitions between VB and $C B$ bound states is represented in Fig. 3 (red curve). When the absorption between VB extended sates and the IB and ladder states are added, the black curve is obtained. It is apparent that the extended states affect the absorption curve very slightly and that our assumption in $[16,17]$, that only the transitions between bound
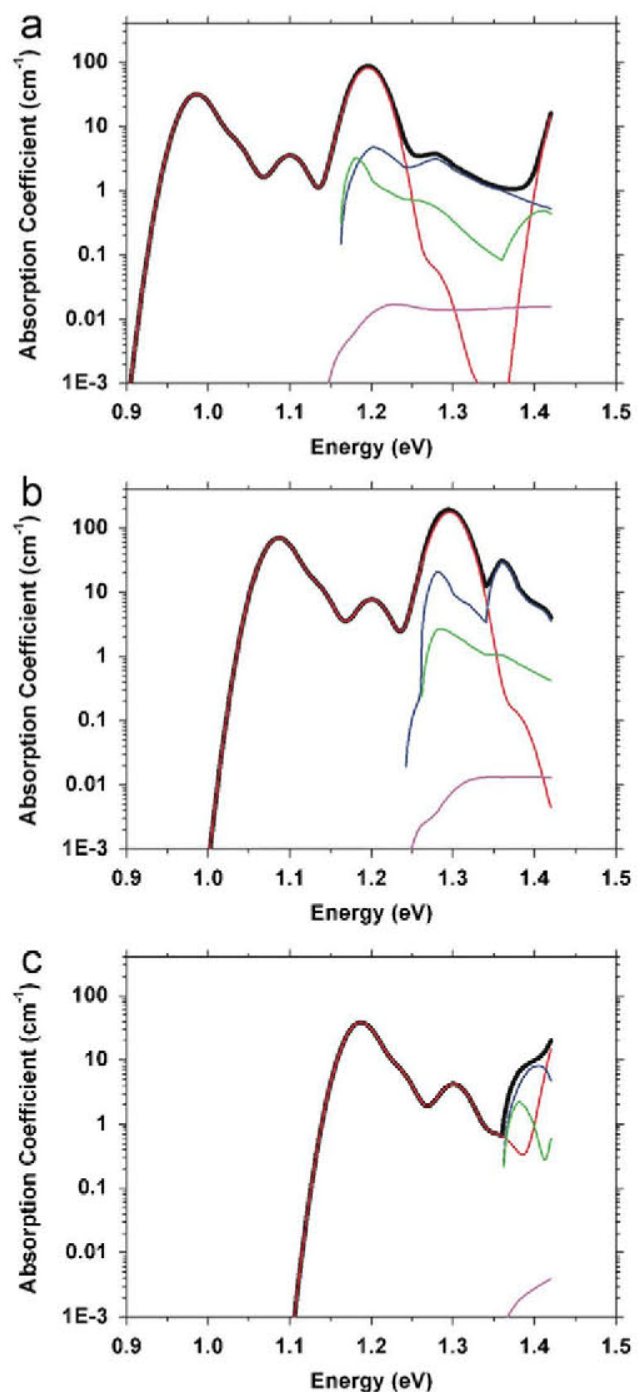

Fig. 4. Absorption coefficient for the transitions from the VBs and the bound states of the CB. Red: from the OE bound states. Blue: from the 1E states. Green: from the 2E states. Purple: from the 3E states. Black: sum of all the preceding. (a) to cb (111), (b) to $\mathrm{cb}(121) /(221)$ and (c) to $\mathrm{cb}(221)$ (For interpretation of the references to color in this figure legend, the reader is referred to the web version of this article.)

states is important, is correct. The agreement between the calculated and the measured $\mathrm{QE}$, that was a justification of the model, is confirmed by the results of this paper.

The transitions from the bound states of the VB to the virtual bound sates within the $C B$ is represented by the blue curve in Fig. 3. Actually, this constitutes a shrinking of the bandgap due to the QDs and in a wider sense reflects transitions between the VB and the $\mathrm{CB}$. This is so because the hh states are in thermal contact with the whole VB due to the small energy interval between them. The same can be said about the lh states, which are immersed in the hh range of energies. On the other hand, the cb virtual bound states are within the $\mathrm{CB}$ and therefore in thermal equilibrium with it. This shrinkage is visible in Fig. 1 where the hh states, colored in magenta, represent the extent to which the VB enters into the GaAs bandgap.

This bandgap shrinkage does not necessarily imply a substantial reduction of the voltage of the solar cells currently manufactured with this material, as shown in $[25,26]$. In reality, the density of (hh and $\mathrm{lh}$ ) states introduced within the band by the QDs is rather faint (as confirmed by the weak absorption of light). The situation may change if a much bigger density of QDs is introduced. 
Observe also in Fig. 3 that the influence of the extended states is really important beyond $1.38 \mathrm{eV}$. This is the region in which a rather strong absorption is produced, attributed to the wetting layer associated to the manufacturing of the QDs by growth of epitaxial layers of InAs on GaAs in the Stranski Krastanov model. This layer actually forms a quantum well whose absorption is much stronger. No attempt to model the $Q E$ in this region has been made in [16,17].

In this paper, the transitions between the VB bound states and the extended states of the $C B$ have not been studied. We are mainly interested in how the $\mathrm{cb}$ bound states situated within the band are filled with VB electrons by absorption of photons. The transitions between VB bound sates and $\mathrm{CB}$ extended states do not affect the latter mechanism and their calculation is only useful to predict the sub-bandgap $Q E$. In addition, by analogy with the transitions between VB extended states and cb sub-bandgap bound states, it has to be presumed that their role is small as compared to the transitions between bound states represented by the blue curve in Fig. 3.

It is also of interest to bring to this paper a conclusion from another paper [27] related to the transitions between the IB and the ladder sates and the $C B$. In this case, only the transitions between bound states and virtual bound sates are permitted together with the transitions between bound sates and 1E states in the $\mathrm{CB}$. However, the latter is weaker that the former:

If we extend this section to report not only to what we have found in this paper, but also of what we have learned in several years of research on the challenges still remaining to manufacture a practical IB solar cell based on QDs, the first of all is the weak sub-bandgap light absorption. This is mainly due to the small density of QDs existing in the solar cells, presently in the range of $5 \times 10^{15} \mathrm{~cm}^{-3}$. Increases 20 fold present values [16] are at the reach of the today's technology if surfaces with higher crystallographic indices are used as growth substrate to promote a denser nucleation $[28,29]$ and the QD layer separation is reduced from $80 \mathrm{~nm}$, used by us to prevent tunneling, to the $20 \mathrm{~nm}$ commonly used. Tunneling may be prevented in this case using field damping layers, or by employing a wide pitch for the QDs situated in the space charge region and a narrower one for those in the neutral zones. With these values of the IQE of 0.1 for the lowest IB absorption are achievable. This value may be higher if we consider that smaller QDs, like those produced in [28] will increase the light absorption about 2-3 times [30]. Furthermore, the number of QD layers may be increased from the present 40 in our modeled cell to up to 100 achieved by several authors. Finally, photon management techniques may also be added to the solar cell.

In the early stages, much discussion was placed on the photon selectivity that requires that each photon is only absorbed by the transition that allows the highest efficiency [31,32]. This is not an issue at the present time, where the absorption of sub-bandgap photons is weak and few are detracted by the photon flow. Even in a scenario of strong absorption, part of the problem is solved insofar that the photons allowing $\mathrm{VB}-\mathrm{CB}$ transitions may be absorbed by the emitter that is an ordinary semiconductor.

Another challenge is associated with the preservation of the voltage, one of the important claims for the ideal IB solar cell. Present IB solar cells, as most of the solar cells of any type, have their open circuit voltage limited by non-radiative SRH recombination associated to deep recombination centers caused by impurities and imperfections. Their recombination current is controlled by the minority carriers and these depend on the bandgap, a higher bandgap producing a lower recombination current. However the IB solar cells operating at room temperature present an effective bandgap for this recombination that is the energy difference between the IB-level and the VB $[3,33]$, which is smaller than the bandgap of the cell without the QDs. Therefore the SRH recombination is stronger and the voltage is smaller. The reason for this reduced bandgap is that the IB is in thermal contact to the actual $C B$ through the ladder states described in this paper. When the temperature is decreased this thermal contact (through thermal photons and phonons) is reduced and the IB solar cell may recover [34] very high open circuit voltage. Good quality IB solar cells may counteract this shortcoming, as has been proven in practice [35].

The IB-CB thermal contact is reduced in larger bandgap solar cell as those with barrier material of GaAlAs [36]. Furthermore, smaller QDs may remove the ladder states and therefore reduce the thermal contact, besides increasing the illumination photon absorption [27].

A longer review is presented in [3].

\section{Acknowledgements}

This paper is partially sponsored by the European Commission NGCPV (283798) and the Madrid Regional Government grant NUMANCIA-2 (S2009/ENE1477).

\section{References}

[1] A. Luque, A. Martí, Increasing the efficiency of ideal solar cells by photon induced transitions at intermediate levels, Physical Review Letters 78 (1997) 5014-5017.

[2] A. Luque, A. Martí, A metallic intermediate band high efficiency solar cell, Progress in Photovoltaics: Research and Applications 9 (2001) 73-86.

[3] A. Luque, A. Marti, C. Stanley, Understanding intermediate-band solar cells, Nature Photonics 6 (2012) 142-152.

[4] K.M. Yu, W. Walukiewicz, J. Wu, W. Shan, J.W. Beeman, M.A. Scarpulla, O.D. Dubon, P. Becla, Diluted II-VI oxide semiconductors with multiple band gaps, Physical Review Letters 91 (2003) 246403-246404.

[5] W. Wang, A.S. Lin, J.D. Phillips, Intermediate-band photovoltaic solar cell based on ZnTe:O, Applied Physics Letters 95 (2009) 011103.

[6] N. Lopez, L.A. Reichertz, K.M. Yu, K. Campman, W. Walukiewic, Engineering the electronic band structure for multiband solar cells, Physical Review Letters 106 (2011) 028701.

[7] A. Martí, L. Cuadra, A. Luque, Quantum dot intermediate band solar cell, in: IEEE (Ed.) Proceedings of the 28th IEEE Photovoltaics Specialists Conference, New York, 2000.

[8] A. Luque, A. Martí, C. Stanley, N. López, L. Cuadra, D. Zhou, A. Mc-Kee, General equivalent circuit for intermediate band devices: potentials, currents and electroluminescence, Journal of Applied Physics 96 (2004) 903-909.

[9] S.M. Hubbard, C.D. Cress, C.G. Bailey, R.P. Raffaelle, S.G. Bailey, D.M. Wilt, Effect of strain compensation on quantum dot enhanced GaAs solar cells, Applied Physics Letters 92 (2008) 123512

[10] C.G. Bailey, D.V. Forbes, R.P. Raffaelle, S.M. Hubbard, Near $1 \mathrm{~V}$ open circuit voltage InAs/GaAs quantum dot solar cells, Applied Physics Letters 98 (2011) 163105.

[11] Y. Okada, R. Oshima, A. Takata, Characteristics of InAs/GaNAs strain-compensated quantum dot solar cell, Journal of Applied Physics 106 (2009) 024306.

[12] S.A. Blokhin, A.V. Sakharov, A.M. Nadtochy, A.S. Pauysov, M.V. Maximov, N.N. Ledentsov, A.R. Kovsh, S.S. Mikhrin, V.M. Lantratov, S.A. Mintairov, N.A. Kaluzhniy, M.Z. Shvarts, AlGaAs/GaAs Photovoltaic Cells with an Array of InGaAs ODs, Semiconductors 43 (2009) 514-518.

[13] D. Zhou, P.E. Vullum, G. Sharma, S.F. Thomassen, R. Holmestad, T.W. Reenaas, B.O. Fimland, Positioning effects on quantum dot solar cells grown by molecular beam epitaxy, Applied Physics Letters 96 (2010) 083108.

[14] V. Popescu, G. Bester, M.C. Hanna, A.G. Norman, A. Zunger, Theoretical and experimental examination of the intermediate-band concept for strainbalanced (In,Ga)As/Ga(As,P) quantum dot solar cells, Physical Review B 78 (2008) 205321.

[15] E. Antolín, A. Marti, C.D. Farmer, P.G. Linares, E. Hernández, A.M. Sánchez, T. Ben, S.I. Molina, C.R. Stanley, A. Luque, Reducing carrier escape in the $\ln$ As/ GaAs quantum dot intermediate band solar cell, Journal of Applied Physics 108 (2010) 064513.

[16] A. Luque, A. Marti, E. Antolín, P.G. Linares, I. Tobías, I. Ramiro, E. Hernandez, New Hamiltonian for a better understanding of the quantum dot intermediate band solar cells, Solar Energy Materials \& Solar Cells 95 (2011) 2095-2101.

[17] A. Luque, A. Mellor, E. Antolin, P.G. Linares, I. Ramiro, I. Tobias, A. Marti, Symmetry considerations in the empirical k.p Hamiltonian for the study of intermediate band solar cells, Solar Energy Materials and Solar Cells 103 (2012) 171-183.

[18] S. Datta, Quantum Phenomena, Addison WesleyReading, Massachusetts, 1989. 
[19] A. Luque, A. Mellor, I. Tobías, E. Antolín, P.G. Linares, I. Ramiro, A. Martí, Virtual-bound, filamentary and layered states in a box-shaped quantum dot of square potential form the exact numerical solution of the effective mass Schrödinger equation, Physica B: Condensed Matter 413 (2013) 73-81.

[20] R. Strandberg, T.W. Reenaas, Photofilling of intermediate bands, Journal of Applied Physics 105 (2009) 124512

[21] A. Luque, A. Marti, E. Antolín, P.G. Linares, I. Tobias, I. Ramiro, Radiative thermal escape in intermediate band solar cells, AIP Advances 1 (2011) 022125.

[22] A. Luque, A. Marti, E. Antolin, P. Garcia-Linares, Intraband absorption for normal illumination in quantum dot intermediate band solar cells, Sola Energy Materials \& Solar Cells 94 (2010) 2032-2035.

[23] A. Luque, A. Marti, A. Mellor, D.F. Marron, I. Tobias, E. Antolín, Absorption coefficient for the intraband transitions in quantum dot materials, Progress in Photovoltaics (2012), http://dx.doi.org/10.1002/pip.1250.

[24] A. Messiah, Mécanique Quantique, Dunod, Paris, 1960.

[25] I. Tobías, A. Luque, A. Martí, Numerical modeling of intermediate band solar cells, Semiconductor Science and Technology 26 (2011) 014031.

[26] A. Luque, E. Antolín, P.G. Linares, I. Ramiro, A. Mellor, I. Tobías, A. Martí, Interband optical absorption in quantum well solar cells, Solar Energy Materials \& Solar Cells 112 (2013) 20-26.

[27] A. Mellor, A. Luque, I. Tobias, A. Marti, The influence of quantum dot size on the sub-bandgap intraband photocurrent in intermediate band solar cells, Applied Physics Letters 101 (2012) 133909.

[28] K. Akahane, T. Kawamura, K. Okino, H. Koyama, S. Lan, Y. Okada, M. Kawabe, M. Tosa, Highly packed InGaAs quantum dots on GaAs(311)B, Applied Physics Letters 73 (1998) 3411-3413.
[29] K. Akahane, N. Ohtani, Y. Okada, M. Kawabe, Fabrication of ultra-high density InAs-stacked quantum dots by strain-controlled growth on $\ln P(311) B$ substrate, Journal of Crystal Growth 245 (2002) 31-36.

[30] A. Mellor, A. Luque, I. Tobias, A. Marti, A numerical study into the influence of quantum dot size on the sub-bandgap interband photocurrent in intermediate band solar cells, AlP Advances 3 (2013) 022116-022117.

[31] A.S. Brown, M.A. Green, R.P. Corkish, Limiting efficiency for a multi-band solar cell containing three and four bands, Physica E 14 (2002) 121-125.

[32] L. Cuadra, A. Marti, A. Luque, Influence of the overlap between the absorption coefficients on the efficiency of the intermediate band solar cell, IEEF Transactions on Electron Devices 51 (2004) 1002-1007.

[33] A. Luque, P.G. Linares, E. Antolín, I. Ramiro, C.D. Farmer, E. Hernández, I. Tobías, C.R. Stanley, A. Martí, Understanding the operation of quantum dot intermediate band solar cells, Journal of Applied Physics 111 (2012) 044502.

[34] P.G. Linares, A. Marti, E. Antolin, C.D. Farmer, I. Ramiro, C.R. Stanley, A. Luque, Voltage recovery in intermediate band solar cells, Solar Energy Materials and Solar Cells 98 (2012) 240-244.

[35] C.G. Bailey, D.V. Forbes, S.J. Polly, Z.S. Bittner, Y. Dai, C. Mackos, R.P. Raffaelle, S. M. Hubbard, Open-circuit voltage improvement of InAs/GaAs quantum-dot solar cells using reduced InAs Coverage, IEEE Journal of Photovoltaics 2 (2012) 269-275.

[36] I. Ramiro, E. Antolin, M.J. Steer, P.G. Linares, E. Hernandez, I. Artacho, E. Lopez, T. Ben, J.M. Ripalda, S.I. Molina, F. Briones, C.R. Stanley, A. Marti, A. Luque, InAs/ AlGaAs quantum dot intermediate band solar cells with enlarged subbandgaps, in: Proceedings of 38th IEEE Photovoltaic Specialists Conference 2012 (PVSC), 2012, pp. 000652-000656. 